\title{
A Review of Mobile Robotic Telepresence
}

\author{
Annica Kristoffersson, Silvia Coradeschi, and Amy Loutfi \\ Center of Applied Autonomous Sensor Systems, Örebro University, Fakultetsgatan 1, 70182 Örebro, Sweden \\ Correspondence should be addressed to Annica Kristoffersson; annica.kristoffersson@oru.se
}

Received 30 August 2012; Revised 29 December 2012; Accepted 5 February 2013

Academic Editor: Eva Cerezo

Copyright (C) 2013 Annica Kristoffersson et al. This is an open access article distributed under the Creative Commons Attribution License, which permits unrestricted use, distribution, and reproduction in any medium, provided the original work is properly cited.

\begin{abstract}
Mobile robotic telepresence (MRP) systems incorporate video conferencing equipment onto mobile robot devices which can be steered from remote locations. These systems, which are primarily used in the context of promoting social interaction between people, are becoming increasingly popular within certain application domains such as health care environments, independent living for the elderly, and office environments. In this paper, an overview of the various systems, application areas, and challenges found in the literature concerning mobile robotic telepresence is provided. The survey also proposes a set terminology for the field as there is currently a lack of standard terms for the different concepts related to MRP systems. Further, this paper provides an outlook on the various research directions for developing and enhancing mobile robotic telepresence systems per se, as well as evaluating the interaction in laboratory and field settings. Finally, the survey outlines a number of design implications for the future of mobile robotic telepresence systems for social interaction.
\end{abstract}

\section{Introduction}

Telepresence [1] is about the sense of being in another environment. In particular, robotic telepresence offers the means to connect to a remote location via traditional telepresence with the added value of moving and actuating in that location. A facet of robotic telepresence is social robotic telepresence where the primary aim of the system is to foster a social interaction between individuals.

In this paper, we provide the first literature review of a subset of social robotic telepresence systems whose focus is on mobility. Such systems concentrate primarily on enabling social interaction via a video conferencing system with the added functionalities of moving/steering the system to various locations. Adopted from [2,3], we refer to this subset of social robotic telepresence devices as mobile robotic telepresence (MRP) systems. Typical MRP systems are characterized by an LCD screen, a web camera, a microphone, and speakers allowing communication between two parties. The units can be moved around by a user who is not situated at the robot site.

Today, the field of mobile robotic telepresence is in rapid expansion, with an increasing amount of commercial systems available and research efforts in the field [4-6]. The published research spans from issues on navigation and immersion to evaluations in office and health care environments. So far, however, there is a lack of a comprehensive review of MRP systems in the literature, and the primary aim of this work is to provide an overview of the systems available, their intended use, and the core research directions which have appeared in the literature. This paper also aims to summarize the various works by providing a reflection of the lessons learned from these various research initiatives. In the literature, a variety of terms have been used to refer to the system and the users. This paper therefore also proposes to adopt a specific terminology using the following definitions.

Mobile robotic telepresence (MRP) systems are characterized by a video conferencing system mounted on a mobile robotic base. The system allows a pilot user to move around in the robot's environment. The primary aim of MRP systems is to provide social interaction between humans. The system consists of both the physical robot (sensors and actuators) and the interface used to pilot the robot.

A Pilot user is a person who remotely connects to the robot via a computer interface. The pilot who is embodied in the MRP system can move around in the 
environment where the robot is located and interact with other persons.

\begin{abstract}
A Local user is the user that is being situated at the same physical location as the robot. Local users are free to move around while interacting with the pilot user who is visiting them via the robot.
\end{abstract}

Local environment is the environment in which the robot and the local user are situated.

The paper is organized as follows. Section 2 outlines a list of MRP systems which are later referred to in the paper. In Section 2, focus is placed solely on the MRP systems' design and technical specifications. Section 3 contextualizes these systems by dividing them into a number of application areas where focus is placed on specific design choices that are directed towards the application needs. Section 4 provides an overview of the various methods used when evaluating MRP systems, including both technical evaluations and user feedback. Section 5 outlines a number of design implications to consider for developers of future MRP systems based on the results of the evaluations and experiences from using systems in the different application areas. Finally, Section 6 provides a future outlook of the field of MRP systems.

\section{MRP Systems Design}

MRP systems vary in design and functionality often depending directly on their intended use and application. In this section, we briefly describe the hardware and software specification of the most common MRP systems found in the literature (see Table 1). Although some of the manufacturers of the robots provide public price tags, the field is undergoing rapid expansion. The price tags are therefore expected to change over time.

Among the first of the MRP systems was PRoP $[7,8]$; see Figure 1(a). The basic construction of PRoP consisted of a mobile robotic base, an LCD screen, camera, microphone, a robot hand/arm hardware with a 2 DOF pointer, and a laser pointer attached to its tip for simple gesturing. As described in [8], pilots have the ability to move the robot as well as zoom, pan, and tilt the head. For navigation, a keyboard and a joystick are needed. The goal with PRoP was to allow the pilot to fully immerse in real remote spaces and a number of services such as a visual scrapbook logging interactions and movements were envisioned [8].

Since ProP, many similar types of MRP systems have emerged varying only slightly in functionality and design. Giraff, [9], see Figure 1(b), is a human-height MRP system. It comes with a $14.1^{\prime \prime}$ tiltable standing screen mounted on a nonadjustable pole attached to a mobile base. The web cam provides the pilot, who connects from a PC, with a wideangle view of the local environment. The construction and design of Giraff is highly motivated by its intended use in a home environment. Thus the Giraff robot, with its heavy base, can clear thresholds and obstacles with minimum risk of tipping. Driving the robot can be done by using a mouse or touchpad. The approximate tentative trajectory is drawn as a red line on a video panel. When the left mouse button is pressed and held, the line transitions to green and the robot starts moving. The robot's direction and speed are controlled by the orientation and length of the line, respectively. The robot's head tilt can be adjusted at any point during driving. Motivated again by its application for home use, Giraff faces the wall when positioned in the docking station. Further, a database is used to handle access rights between pilots and robots. In the normal usage, the local user has to respond to the "calls" but an emergency call access level exists.

QB [10], see Figure 1(c), has a manually adjustable height and its base has two dynamically balancing wheels compared to the more common four wheels for MRP systems. The QB comes with two cameras: one five megapixel nontilt front facing camera and one down facing camera for navigation. Deictic referencing is done with a laser pointer. The system can be piloted from any browser on Windows and Macintosh [11]. The device is suited for office environments.

Texai has an alpha prototype [12], see Figure 1(d), with a $19^{\prime \prime}$ touch screen and a pan-tilt, wide-angle web camera mounted on a mobile base equipped with a bumper. It has two laser-range finders. Fully charged, the Texai can run for eight hours. It leverages on the open source robot operative software (ROS) [13] and commercially available video conferencing software. To preserve privacy, the screen is black when no pilot is using the MRP system. While the research on Texai continues, a new company Suitable Technologies [14] has been created in order to take the product to the market. During the fall of 2012, the company released the new commercial human-height MRP system called Beam, see Figure 1(e). The system features include a $17^{\prime \prime}$ screen, a six-microphone array enabling pilot users to localize directions of sound, two wide-angle HD cameras (one front facing and one down facing), and digital zoom. The battery time is reported to be long enough to last a full working day. The Beam docks backwards and LEDs are used to show it is docked. The system can be piloted from Windows and Macintosh.

VGo [15], see Figure 1(f), can be piloted from both Windows and Macintosh. The navigation is done by "click and go" buttons and arrow keys. The pilot can take snapshots of the local environment as well as zoom up to 5 times in the video. The VGO can be used on Verizon 4G LTE and WiFi. It has a fixed height and comes with two battery options, 6 or 12 hours.

PEBBLES [16], see Figure 1(g), has been designed to allow young hospitalized children to attend school embodied in a MRP system and has two versions: one for elementary school and one for high school students (where the latter is smaller in order to adjust to the rotary schedule typical for high school). The high school version also supports two screens allowing for participation in group activities, one screen for communication and one for desktop- and file sharing. Both platforms are characterized by the typical wheeled robotic device with a simple arm-like attachment which enables the pilot user to perform a gesture similar to a hand raise. Pan and tilt units are also present on the head of the robot.

A current trend is the use of smartphone technology and/or tablets as integral components in the MRP system design. MantaroBot Classic and TeleMe [19], see Figures 1(h) 
TABLE 1: An overview of common MRP systems appearing in the literature.

\begin{tabular}{|c|c|c|c|c|c|c|}
\hline MRP system & Commercial & $\begin{array}{l}\text { Intended } \\
\text { application } \\
\text { area }\end{array}$ & $\begin{array}{l}\text { Adjustable } \\
\text { height }\end{array}$ & Manipulation/expressions & Navigation/sensors & $\begin{array}{l}\text { Selected } \\
\text { references }\end{array}$ \\
\hline PRoP & No & Research & No & $\begin{array}{l}\text { Laser pointer, } 2 \mathrm{DOF} \\
\text { hand/arm }\end{array}$ & No & {$[7,8]$} \\
\hline Giraff & Yes & Elderly & No & No & No & [9] \\
\hline QB & Yes & Office & Yes & Laser pointer & $\begin{array}{l}\text { Motion sensors for platform } \\
\text { stabilization }\end{array}$ & {$[10,11,17]$} \\
\hline Texai & No & Office & No & No & Laser-range finders, bumper & {$[12]$} \\
\hline Beam & Yes & Office & No & No & No & {$[14]$} \\
\hline VGo & Yes & Office & No & $\begin{array}{l}\text { Handheld remote for local } \\
\text { control }\end{array}$ & Cliff sensor & {$[15,17,18]$} \\
\hline PEBBLES & No & School & No & Hand & No & {$[16]$} \\
\hline $\begin{array}{l}\text { MantaroBot } \\
\text { Classic }\end{array}$ & Yes & Office & Yes & Laser pointer & $\begin{array}{l}\text { Infrared obstacle detection, tip } \\
\text { detection using three accelerometers }\end{array}$ & [19] \\
\hline $\begin{array}{l}\text { MantaroBot } \\
\text { TeleMe }\end{array}$ & Yes & Office & No & Laser pointer & $\begin{array}{l}\text { Infrared obstacle detection, tip } \\
\text { detection using three accelerometers }\end{array}$ & [19] \\
\hline Double & Yes & Unspecified & $\begin{array}{l}\text { Yes } \\
\text { motorized }\end{array}$ & No & $\begin{array}{l}\text { Gyroscope and accelerometer for } \\
\text { balance. Kick-stands when static. }\end{array}$ & [20] \\
\hline mObi & No & Research & No & No & Kick-stands for safety. & {$[21]$} \\
\hline Jazz Connect & Yes & Office & No & No & $\begin{array}{l}\text { Obstacle detection. } 8 \text { ultrasonic } \\
\text { sensors, } 4 \text { IR sensors, and high-end } \\
30 \mathrm{~m} \text { range telemetric laser for } \\
\text { autonomous navigation (optional) }\end{array}$ & {$[22,23]$} \\
\hline iRobot Ava & Yes & Healthcare & Yes & Yes & $\begin{array}{l}\text { Laser, Sonar, } 2 \mathrm{D} / 3 \mathrm{D} \text { imaging for } \\
\text { autonomous navigation, Cliff sensor, } \\
\text { and contact bumper. Omnidirectional } \\
\text { navigation }\end{array}$ & {$[24]$} \\
\hline $\begin{array}{l}\text { 9th Sense } \\
\text { Helo and Telo }\end{array}$ & Yes & Unspecified & No & No & $\begin{array}{l}\text { No, but ports for peripherals are } \\
\text { available. }\end{array}$ & {$[25]$} \\
\hline RP-7 & Yes & Healthcare & No & No & $\begin{array}{l}\text { Omnidirectional navigation. Obstacle } \\
\text { detection via }+30 \text { infrared sensors }\end{array}$ & {$[26,27]$} \\
\hline MeBot & No & Research & No & $\begin{array}{l}3 \text { DOF arms and } 3 \text { DOF } \\
\text { neck }\end{array}$ & Obstacle and cliff detection & {$[28]$} \\
\hline
\end{tabular}

and 1(i), are two MRP systems where the TeleMe in particular supports a smart phone or tablet as the head of the robot. Skype is used for video conferencing and the pilots can move the robots around, pan and tilt the screen, and adjust the height remotely via a Skype plugin.

Similarly, using smartphone technologies to control MRP systems is of interest in order to promote access anywhere for the pilot operators. In this light, new interfaces such as touchscreens can make the task of teleoperation harder, and therefore, semi-autonomous functions are increasingly useful on MRP systems. For example, Jazz Connect [22], see Figure 1(j), can be accessed from smartphones or computers via a web-based interface. The robot turns in the direction on which the pilot points and clicks in the video image. A "go to" control is available directly in the video image. A warning is given via the pilot interface when the pilot is driving close to obstacles. Automatic docking is possible when the Jazz Connect is within $2 \mathrm{~m}$ from the docking station. The system offers flexibility in that developers can use the Gostai Suit to reuse code from other Urbi compatible robots [23] and to develop new widgets to control the robot. iRobot Ava [24] follows a similar design strategy dubbed "Head Agnostic Design" where tablets, displays, and phones are interchangeable components for the display on the MRP system as well as the interaction device for the pilot user (see Figure $1(\mathrm{k})$ ). Likewise, the devices come with an extensive sensor suite and functions, for example, automized docking and adjustable height. Similarly, 9th Sense [25] introduced Helo and Telo (Figures $1(\mathrm{l})$ and $1(\mathrm{~m})$ ), two robots that can be operated directly via a computer keyboard when connected to the robots via Skype. While both systems are customizable in that they allow for sensor and USB plugins, Helo is more compact and uses a Samsung Galaxy Tab as a head.

Also leveraging on the use of tablets for the screen, several of MRP systems utilize standard solutions for the robotic base. The commercially available Segway-like light-weight Double [20], see Figure 1(n), moves about on two wheels and uses a gyroscope and accelerometers to stay in balance. While not moving, kickstands are used to lessen the consumed power. The height of the robot can be adjusted to any height 


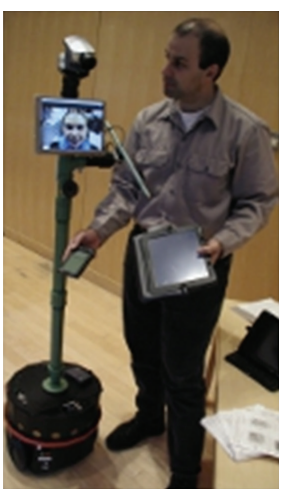

(a)

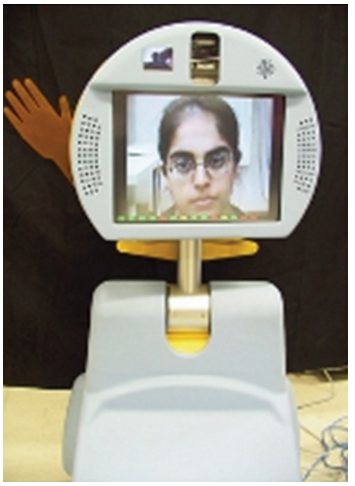

(g)

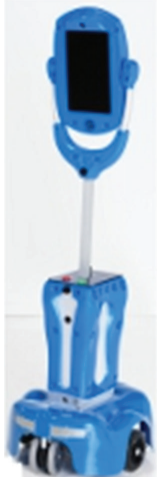

(b)

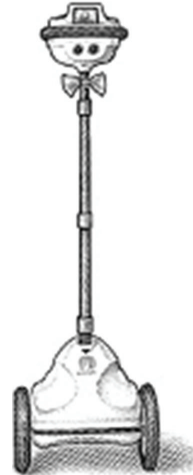

(c)

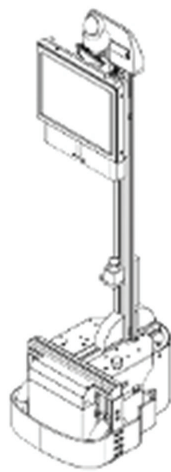

(d)

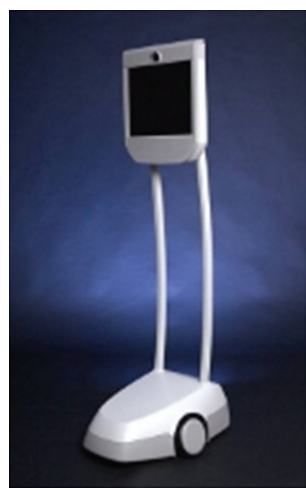

(e)

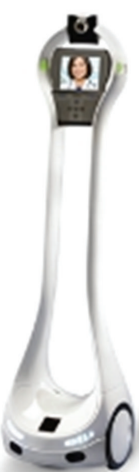

(f)

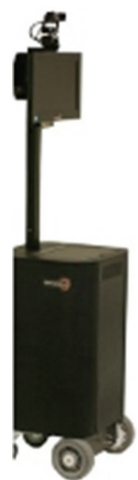

(h)

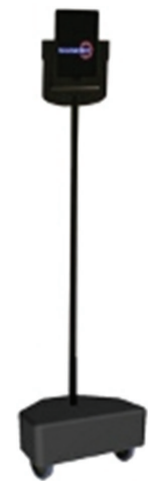

(i)

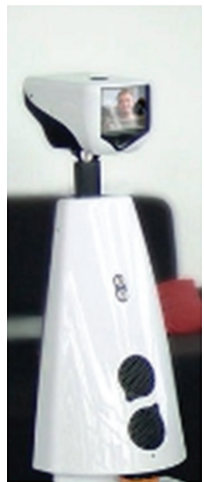

(j)

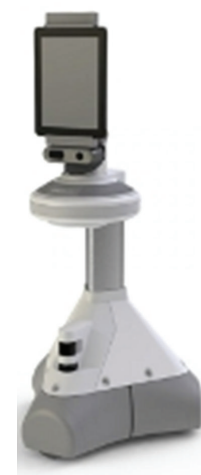

(k)

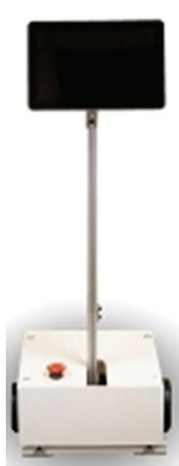

(1)

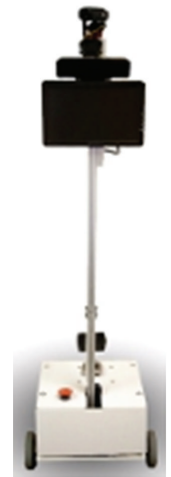

(m)

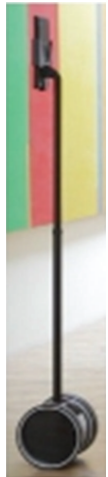

(n)

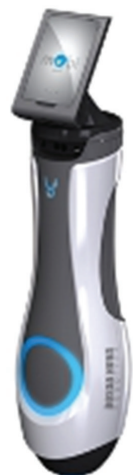

(o)

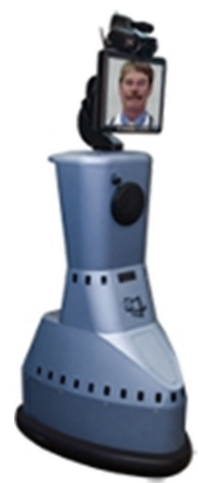

(p)

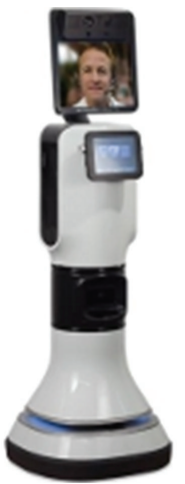

(q)

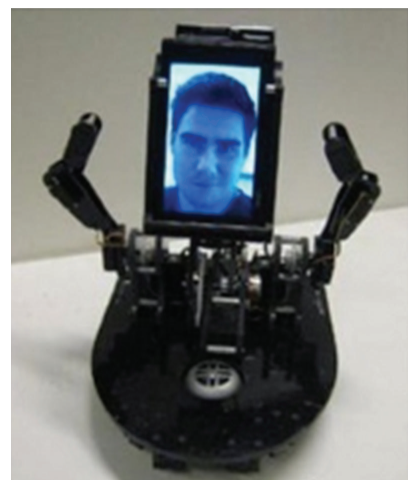

(r)

Figure 1: (a) PRoP, (b) Giraff, (c) QB, (d) Texai, (e) Beam, (f) VGo, (g) PEBBLES, (h) MantaroBot Classic, (i) MantaroBot TeleMe, (j) Jazz Connect, (k) iRobot Ava, (l) 9th Sense Helo, (m) 9th Sense Telo, (n) Double, (o) mObi, (p) RP-7, (q) RP-VITA, and (r) MeBot.

between $100 \mathrm{~cm}$ and $150 \mathrm{~cm}$. The robot uses an iPad as a head and it is remotely controlled through an iPad app that the pilot uses to connect to the robot. The first MRP system using a ballbot technology (mObi [21], see Figure 1(o)) is expected to be available for researchers and developers during 2013. The robot balances and moves about on a ball. Also this system uses kickstands as an automatic safety mechanism. The robot head has a docking station for a tablet and is expected to make use of 3D depth sensors.

While many of the above systems have been made available recently on the market, one of the more established MRP systems, particularly for the North American market, is the RP-7 (see Figure 1(p)) by InTouch Health [26]. Geared towards operational use in primary and secondary care units, the RP-7 design is suitable for a clinical setting conforming to medical regulations as set by the FDA (US Food and Drug Administration). RP-7 features two high-resolution cameras and a proprietary video conferencing solution for two-way communication. In the summer of 2012, InTouch Health together with iRobot unveiled the new RP-VITA (Figure 1(q)) with improved navigation capabilities, real time clinical access to patient data, and an iPad interface enabling quick and easy access to pilots navigating the RP-VITA.

All of the above mentioned systems have some basic ingredients in common. These include a typically nonanthropomorphic appearance and design, where only a limited 
subset of human skills is possible (e.g. pan-tilt, simple gesticulation) and two-way audio and video communication. This approach differs fundamentally from other types of telepresence systems that involve an anthropomorphic design such as an android like platform. Though this study is restricted to MRP systems in the nonanthropomorphic sense, Figure 2 shows some of the more well researched tele-android systems which aim primarily in recreating a more realistic face-toface remote human experience. Geminoid HI-2 [29], shown in Figure 2(a), is the first teleoperated android that very closely resembles a human, while Telenoid (see Figure 2(c)) has opted to adopt minimal human likeness in order to convey a human but unidentifiable presence that is perceived without specific gender or appearance [30,31]. Also other systems which do not fall within the MRP system definition in this paper include the floating avatar (Figure 2(e)) that integrates a blimp with a virtual avatar. The pilots can control the blimp from remote and communicate with local users by transmitting their facial images over the network [32]. Another example is the $\mathrm{TRiC}_{\text {mini }}$ (Figure 2(f)), a doll size, low weight, baby-like robot described in [33]. While $\mathrm{TRiC}_{\text {mini }}$ is operated by the pilot, the local user can lift the robot in case there are barriers and dock it into any electric socket. The local user may also easily personalize the robot, for example, by customized clothing.

Finally, there are a number of MRP systems which are closer to research prototypes than marketable products. This includes the TeCaRob project [34] which aimed to provide a customized and on-demand remote assistance. The platform had four subsystems: (1) a robotic platform consisting of a mobile base, arms, and arm extremities, (2) sensors including cameras, thermostats, on/off sensors, location tracking, 3D rendering of end users, and so forth, (3) an interaction platform to socialize, and (4) a communication platform ensuring continuity, security, and privacy. Another example is MeBot (Figure 1(r)) which allows pilots to convey social expressions by performing gestures on the robot. Having undergone several iterations in its design, the final version, MeBot V4 [28], has a mobile and portable base that is capable to avoid obstacles and detect edges. It has two 3 DOF arms (shoulder rotation, shoulder extension, and elbow extension) and its face is mounted on a $3 \mathrm{DOF}$ neck allowing MeBot to pan, tilt, and move forward/backward. The robot mimics the pilot's head movements by using a head pose and orientation estimation API, while the arm movements are directly controlled by the pilot who adjusts the joints on a passive model of the robot. A $3 \mathrm{D}$ mouse is used to move the robot in relation to its current location and its pointer is visualized on an overhead display that also contains sensor data. The pilot user observes the local environment through a custom built screen that has a camera embedded in the middle to aid the users in establishing eye contact.

\section{Application Areas for MRP Systems}

While MRP systems are suitable for a range of applications, there are a number of specific challenges depending on the domain of use. Of the possible applications for MRP systems, three are particularly dominant in the literature: office environments, health care, and aging in place (the ability to live safely, independently, and comfortable in one's own home regardless of age, income, or ability level) for elderly. A possible fourth area which is gaining attention is usage of MRP systems for school environments. The following section describes the use of MRP systems delimited by application area.

3.1. MRP Systems for Office Environments. In societies where the geographic distance between teams who are cooperating is increasing, a set of different MRP systems (QB, Texai, and VGo) has been tested in office environments. The MRP systems allow remote coworkers to visit their local coworkers and participate in formal as well as informal meetings. Promotion of these MRP systems derives from the gain to decrease the amount of travelling for the employees and also to allow immediate access to another site where the employees are needed; meanwhile it also decreases the costs for travelling for the companies. An important factor for successful collaborations is the ability to interact informally. However, typically such communication is brief and not scheduled [3]. Further, such communication requires communication channels that are interactive and expressive [3, 35-37]. Already in 2002 [38], a MRP system was described which allowed pilots to participate in meetings in remote locations. Further developments of the system were presented in $[39,40]$ and a large segment of the research has been performed at companies in the United States of America, for example, [3, 41, 42].

An office environment poses a number of challenges particular for MRP system usage. One challenge is to understand how social norms common in the workplace are affected when interaction between colleagues occurs via MRP systems. Social norms using the Texai as a shared resource among remote coworkers to drive through and communicate with local workers at three different workplaces in the San Francisco Bay area, CA, USA, were examined in [3]. By performing interviews, observations, and surveys with people after 2-18 months of experience of usage of MRP systems, the authors found that the pilots and local users worked almost as if the pilots were there physically. Similarly to [42], they found that the Texai supported informal communications. Before introducing the Texai to colleagues at the company which developed it, the company used the telephone and video conferencing technology to interact with remote coworkers. This often resulted in the remote coworker being left out of meetings and important decision taking [3]. After surveying users it was found that the MRP system was perceived as useful and effective. It was used for a range of activities including impromptu meetings and planned meetings. Since most informal communication occurs in hallways, this had implications for the placement of docking stations. Docking stations were strategically placed in high traffic areas and eating areas while still being close to meeting rooms. Depending on whether the pilot user had its own office desk, the pilot typically parked in the lab or at his/her own office desk. Local users dropped by the office desk in case they wanted to communicate. The system was reported to give more independence to the remote pilot user. However, pilot users 


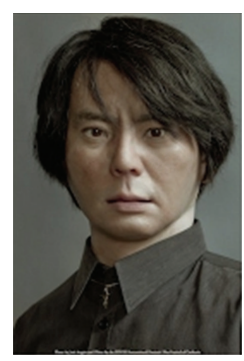

(a)

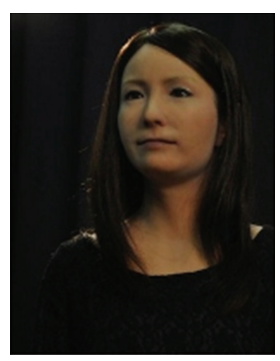

(b)

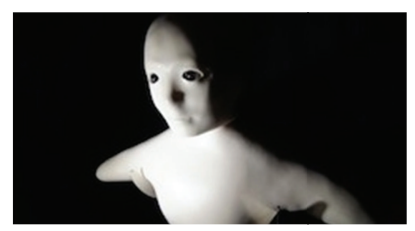

(c)

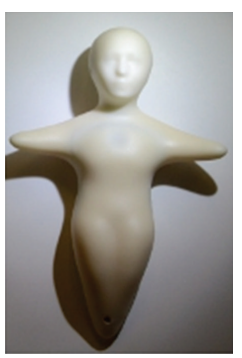

(d)

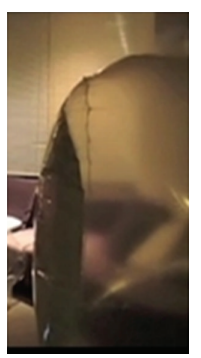

(e)

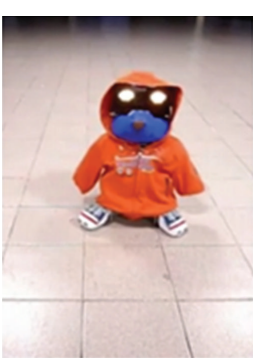

(f)

Figure 2: (a) Geminoid HI-2, (b) Geminoid F, (c) Telenoid, (d) Elfoid, (e) Floating avatar, and (f) TRiC $_{\text {mini }}$.

reported that it was difficult to focus on the social interaction while moving around with locals in hallways. Due to the MRP systems being a shared resource, it was found that an identification system of who is embodying the Texai was needed. In [41] an analysis of the results from five different studies with QB and VGo performed at Google, Mountain View, California, USA, was presented. The authors grouped the results into a set of guidelines regarding different aspects of MRP systems: video, audio, user interface, physical features, autonomous navigation, and social considerations, that is provision of appropriate occupance awareness based on performance and user feedback. How people make sense of a MRP system in an office context was studied in [43] that analyzes what different metaphors people (locals and pilots) used during an 8-week field trial in an effort to shed on light whether the MRP system was treated more like human or more like a machine. Also, the authors of [3] discussed usage norms drawn from other technologies, for example, "hanging up" when having concluding a conversation at inappropriate places. Other norms relating to helping the pilot were also observed.

Additional challenges in office environments are related to sound perception and sound disturbance (as many office environments are open workplaces). For example, that both the local user and the remote pilot user could adjust the volume of the Texai was found to be an important factor [3]. The challenge of regulating the speech volume of the pilot was described in [44]. In particular, due to pilots frequently being perceived as loud in local environments, they performed an experiment where they provided the pilot with an attenuated stream of the pilot's own voice and thereby could improve the experience for the local users as the pilot spoke less loud. However, the improvement was only made when the pilots were using headsets and not when using loudspeakers. In order to increase the scene awareness and allow the pilot to obtain a better indication of the number of sound sources and their directions, [45] examined the possibility to enhance the experience of the pilot in terms of improving the sound perception of a local environment. The approach was to integrate Hark and a visualization tool allowing pilots to "see" in which direction a sound originated. In a controlled experiment, it was found that it was possible to localize surrounding sounds at a tolerance of 5 degrees, however, with a degrading performance when the users were closer together.

In general, an office environment like other application areas requires a level of robustness, security, and data integrity. In [42], the QB was used to connect to work from home during one week. The experimenter found safety and reliability issues in the WiFi coverage resulting in frozen images and disconnections leaving him insecure whether the robot continued moving or not. Guizzo [42] highlights the vulnerability to hacker attacks and the issue of having to ask others to reboot the QB for him.

Summarizing the experiences of evaluating MRP systems in office environments, the systems need to support the remote pilot user in the participation in meetings and engaging in informal interaction with the local coworkers. At an office, informal interaction typically occurs in eating areas and in the hallways on the way to (or from) meetings. Therefore, it is important that the MRP systems support interaction when the pilot user is on the move. Since the MRP systems are also used at meetings, it is also important to aid the pilot in percepting sound and to localize the direction from which sounds are coming.

3.2. MRP Systems for Health Care. Extensive evaluations, in particular with the RP-7 (or RP-6), have been done to study the value of MRP systems in health care. The studies, which are still ongoing, show that the use of MRP systems has reduced the length of stay not only after minor invasive surgeries but also at intensive care units (ICU) as well as reduced response times in emergency situations.

3.2.1. Postoperative Care after Minor Invasive Surgery. The postoperative period after minor invasive surgical procedures usually results in a hospital stay lasting between 24 and 72 hours. Patient safety and satisfaction with either only bed-side rounds or added robotic rounds were assessed in [46]. No major or minor morbidities were observed and $2 / 3$ of the patients agreed that telerounds should be part of regular hospital care. The patients also stated that they would rather be seen remotely by their own physician than by another physician. The authors had previously studied patient satisfaction in a similar study [47] in which the patients stated they would feel comfortable with telerounds in future hospital care. Approximately, 75\% thought it should become standard in postoperative management.

The financial impact of telerounds with regards to length of stay was examined in [48]. The mean length of stay among the patients having the extra telerounds was 1.26 days compared to 2.33 days for the patients only having 
bed-side rounds. The readmission rates were low in both groups of patients but even lower among the patients who had telerounds. The extra telerounds resulted in earlier discharge and an increased bed capacity at the hospital.

\subsubsection{Orthopedic Postoperative Care with Longer Hospital} Stay. The patients' and nurses' satisfaction of using telerounds during evening and weekend ward rounds in an orthopedic clinical setting was examined in [49]. Also in this pilot study, patients agreed that the care improved as a result of telerounds and that it should be a part of patient care at hospitals. Patients thought they could communicate easily with their doctor and felt comfortable having telerounds every day. They even said they would be satisfied with only telerounds on the weekends. As found in [46], they prefer to see their own doctor via telerounds than another doctor at their bed sides. The response from the staff correlated with the response from the patients. All patients agreed it should be part of the routine in managing the postoperative patients during which observation is of importance. However, the authors also pointed out disadvantages and key issues, including security and network stability as well as the inability to open doors and move between floors.

3.2.3. Experiences at Intensive Care Units (ICU). Several articles report on improved response time as a result of the deployment of MRP systems. The consultation time at an ICU was reduced especially during "off-hours" in [50]. Patients could be seen within 5 minutes instead of the physician travelling for 40 minutes. Vespa reported on a preliminary study on usage of telerounds in combination with brain monitoring at a neurologic ICU $[51,52]$. The response time decreased and the level of face-to-face contact between physician and patients increased. The effects of physicians using telerounds during evening time and as a response to nursing pages were studied in [53]. The response time for routine and urgent pages changed from $218 \pm 186$ minutes to $9.2 \pm 9.3$ minutes, an important time saving when addressing brain conditions that may be irreversible. Also [54] advocated the usage of MRP systems in stroke care. Round-the-clock access to experts in stroke care could benefit patients and lead to fewer inappropriate transfers to other hospitals, higher alignment to best practices, and so forth. The length of stay at the ICU decreased and resulted in reduced costs for caring [53]. Other uses of the MRP system reported in this study were mentoring nurses, discussions, on admission and discharging as well as treatment protocols.

Experiences of having a MRP system at a surgical ICU and a burn ICU showed that it was used by critical expertise during night time ward rounds and to respond on pager calls in [55]. The families and patients' perception of the use of telerounds for a multidisciplinary team at a surgical ICU unit was investigated in [56]. An identified problem at the unit was that the normal rounds were causing noise and traffic. This process was changed so that the patient had a morning bedside visit and a physical examination by surgical residents and nurse practitioners after which the multidisciplinary team met in a conference room from where they visited the patient via a MRP system and later planned the patient's care. A focused group of the multidisciplinary team would then visit the patient individually to implement the plan. The patients/families were reported to perceive better quality in the care and supported continued use.

3.2.4. Experiences of Use in Surgery and Teaching. The impact of a MRP system during anatomy laboratory sessions on student and surgeon satisfaction was investigated in [57]. With the MRP system, the remote surgeon could navigate the room and the service platform, tilt and pan the screen, and get close-up views by zooming. The students reported they had a positive experience and mostly felt comfortable with the presence of the MRP system. At times they forgot the surgeon was not there physically. Also the surgeon had a positive experience and claimed to at times forget about the physical distance. The mobility was seen as an improvement to usage of existing fixed systems that typically lack face-toface contact with the students. The authors discuss that while simulation allows the performing of hundreds of procedures, being monitored by an expert is always beneficial while doing a new procedure. However, that usually requires that the expert leaves its practice or that the students come to the expert's institution in which the equipment and supporting staff is different from the student's home setting.

The usage of a MRP system in minimally invasive surgery was examined in [58]. A senior surgeon monitored the operation room during a total of five procedures via the MRP system. The surgeon managed to feel immersed in the operating room and effectively communicated with a junior attendee. No surgery was disrupted due to the presence of the MRP system. The senior consultant could easily maneuver the MRP system around the room and had the same view as the one of the operation team and as the system could link in external camera views. The authors point out some possible uses of the system: (1) capture and magnify images and send back to the operational team via the MRP system while explaining significances of the images and planning the appropriate approach in difficult steps, (2) use linkedin video feeds to provide a more thorough consultation, (3) mentoring and consultation worldwide, (4) time sensitive situations during surgery requiring immediate consultation from an expert, and (5) to provide medical expertise to novice surgeons at hospitals in developing countries.

A report on the OTOROB project was provided in [59]. The OTOROB is equipped with a flexible arm that can enhance a physician's vision of the patient as he can move the camera. It is also equipped with instruments essential in orthopedic care. The authors claim that different tasks may require different robots and although the OTOROB is designed with the orthopedic surgeon in mind, a similar pathway could be used when designing systems for other specialties.

Also the potential of using MRP systems in nursing education was investigated in [60]. Ten faculty members involved in different programs were asked to participate in a study in which they would recruit 5-10 students each who they remotely taught a medical-surgical nursing skill with the aid of a simulator and RP-7. The topic was the faculty member's choice. The teaching and debriefing session 
lasted about one hour after which they were asked to fill a questionnaire. Most students and faculty members rated their acceptance as high. Similarly to the results in [57], the students felt as if the faculty members were physically present.

3.2.5. Postoperative Care in Home. A pilot project that runs at a children's hospital in Boston, MA, USA, in which they test if VGo can be used to monitor patients at home after early discharge was reported in [61]. Instead of taking children to the hospital for regular check-ups they are monitored during video consultations by physicians who have never visited the home of the patient.

3.3. MRP Systems for Elderly and Aging in Place. Stay in place aging for an increasing elderly population in western countries and Japan represents a highly suitable application area for MRP systems. MRP systems could serve various functions simultaneously, such as health surveillance, social interaction, and safeguarding. It is possible to equip a MRP system with medical devices, serve as a link for health care professionals, simultaneously collect data about the elderly, and most importantly promote social interaction for a group who is prone to isolation and loneliness. For safeguarding, MRP systems could work as an emergency alert system if a person had fallen and thereby be connected to other devices in a smart home [62]. A number of evaluations of the "Telerobot system" as an MRP device in domestic settings are presented in [63-68].

A characteristic feature of using MRP systems for aging in place, whether in a private home or residential facility, is that the systems are to be used over extensive periods of time. Therefore, typical measures of evaluation such as acceptance and usability are applicable from a longitudinal perspective. Concepts like "use worthiness" [69] become central to the design and use of the system. A second characteristic for this application domain is that the local users are fragile and commonly have a low experience of computer technologies. This implies that any button that the local user may need to push should have an intuitive meaning and be easy to access. A third challenge is that the homes are often crowded with memories of the elder's previously active life as well as of equipment related to the current needs, for example, wheelchairs and walkers, leaving little space for a MRP system both during its idle state and while moving. Further, the environment often has poor lightning which could make the movement of the robot more difficult than, for example, in office environments. The Giraff robot is currently being evaluated over long periods of time with elderly in the ExCITE project [4]. The long term methodology of ExCITE [70] is circular in which the feedbacks collected regularly from the pilots and local users are fed back to the developers in order to enhance the Giraff according to the needs of users. The methodology is somewhat similar to the one proposed in [71] which included prototyping, field testing in residential care settings, assessment of for, example, satisfaction, and further development. Within the scope of the project, a number of experiments have been reported which concern (1) health care professional views on MRP systems and a comparison between junior and senior professionals and (2) measures for successful interaction with real elderly groups that include physiological measures as well as measuring spatial and social presence [72-76].

Also, [2] has performed a set of studies using elderly persons as pilots. It was found that in certain cases, the elderly prefer to pilot a MRP system rather than receiving a visit via the MRP system. Currently, several research initiatives are focusing on increasing the MRP system utility by adding monitoring capabilities to the systems $[77,78]$. In the GiraffPlus project [78], a system is being created that reasons on collected data from environmental and physiological sensors and allows the pilot user to access such data and interact with the elder through Giraff. The system, which is developed in a closed-loop methodology involving various groups of potential users, can be used by elderly in need of monitoring by, for example, nurses, physicians, and physiotherapists at distance. All such user groups are typically unfamiliar with the home environment of the elder and thus there is a need for including a map in the client software for many potential users of this kind of MRP system.

3.4. MRP Systems for School. Few MRP systems have been developed to help children to attend school even when at hospital or during long term illness at home. PEBBLES was tried successfully with elementary school children in Canada and USA. Additional MRP systems used in the class room to achieve interaction with classmates and teachers are R.BOT 100 developed by Moscow's 3Detection Labs [79], VGo [80, 81], and Texai [82]. Also [83] mentioned this possible use of MRP systems along with the reverse use where a teacher could deliver lectures at distant locations.

Three case studies with PEBBLES have shown that ill children could engage in the same task as their class mates, maintain concentration, and communicate [84]. Over time, PEBBLES has technically changed according to results from a set of user evaluations. The different versions are briefly described in [85]. Realizing that light or flashes were insufficient attention devices in PEBBLES I [86], one of new features in PEBBLES 2 [85] was a waveable hand. Although the hand was found to be effective in gaining the teacher's attention, it raised safety concerns. Evaluations also showed that the system was inappropriate for high school students that typically move between classrooms. Further, it was found difficult to transmit the literature to the ill students. Therefore the PEBBLES II.5 was provided with an extra LCD screen attached to the front of the robot. To improve the view for the ill student, an extra camera providing a global view of the classroom was added. In addition, a scanner and printer were installed at both user ends to improve the logistics. To deal with the safety concerns, the hand was shortened, relocated, and made of a soft fabric. A lightweight portable high school version of PEBBLES, with built-in collaboration tools, was reported on in [87]. The system was sufficiently small to be placed on a desk. However, the version had the limitation that it could only be rotated $45^{\circ}$, less than how a human can turn the head. 
3.5. General MRP Systems. Today, the trend seems to go towards building cheap systems that make use of standard hardware and software for robotics (ROS) and video communication (Skype). This has prompted a number of more generic MRP systems without a particular application domain. MITRO is an ongoing research project at Swarmlab [88] that makes use of and contributes to ROS. MITRO incorporates the Microsoft Kinect sensor, uses Skype for video conferencing, and has two cameras (similarly to the $\mathrm{QB}$, one is pointing forward and one downwards). To provide assisted control and augmented telepresence, the robot is able to perform (simultaneous localization and mapping) SLAM, avoid obstacles with a range of sensors, people, and face tracking. Being inspired by [42], a MRP system for about $\$ 1000$ was assembled in [89]. In contrast to the common four wheels on MRP systems, Schneider used only one wheel to level the system. A netbook sitting atop of a camera is mounted on a tripod atop the robotic base. The system automatically halts and tells the pilot on which side the robot hits an obstacle and an ultrasonic sensor is used to measure the distance to the nearest obstacles for each command. A system with multiple off-the-shelf to iRobots attached robots attached to laptops for unconfined video conferencing and contributed with motion planning algorithms that maintains a good frontal view of the local user for as long as possible was introduced in [90]. The WU Telepresence Robot, a low cost robot using off-the-shelf parts and open source software [91], can be ordered online and be assembled within a couple of hours. The WU Telepresence robot uses Ubuntu and ROS.

\section{User Evaluation Studies}

Evaluation of MRP systems is particularly complex as several types of interactions occur simultaneously. The first is human-robot interaction, that is, the interaction which occurs between the local person and the MRP device per se. The second is human-computer interaction which is the interaction that occurs between the pilot user and the desktop application used to connect to the MRP device. The last is human-human interaction which occurs between the two users, local and pilot, of the system via the MRP device. As found in, for example, [3], the burden of moving the MRP system also influences how well a pilot user and local user can interact. In this section, we will discuss a number of techniques to evaluate the interaction and the client interface used to move the MRP systems around that are typically aiming at lowering the burden of the pilot user and thus increasing the possibility for "good" interaction. The results of the studies reported have implications for the design of future MRP systems. These design implications are summarized in Section 5 .

4.1. Quality of Communication Measures. To maintain a fluid and natural interaction, it is important to respond appropriately when being addressed. "Attention is fundamental to the flow of face-to-face conversation," page 1 [92]. Each participant in an interaction project cues where their attention is directed. The cues are interpreted by the other participants to maintain an awareness of the specific participant's attention and to understand its deictic referencing.

Presence is a multidimensional concept [93] and many questionnaires to measure presence exist. Commonly, two of the dimensions are social and spatial presence which are both of particular relevance for MRP systems. Shortly described, spatial presence occurs when a person's perception fails to accurately acknowledge the role of technology that makes it appear as if that he/she is in a remote environment. Social presence, on the other hand, occurs when a person fails to acknowledge the role of the technology in the communication with others.

In evaluations of MRP systems, assessments of attention and presence are often used as a means to compare systems with varied characteristics regarding movability of the screen, camera, and/or the camera's capability of zooming.

The ability to move has been found to have several effects. For example, a turnable display resulted in a higher level of activity, perceived excitement of the speakers, level of attentiveness and engagement, and amount of turns per second during satellite-hub interaction when compared to interaction through a video conferencing system with a static display [92]. The study included sociometric measures, feedback from the pilot of the system, semi-structured, and several individually filled questionnaires. However, the ability to turn the display also came with tradeoffs such as that any of the local users can be turned away from and thereby feel excluded from discussion. An important result from the study is that sociometric measures are more demonstrative than the response on a subjective questionnaire.

The ability to move the camera forward and backward was found to have significant effects on the social presence when comparing five different conditions: fixed, rotatable, movable but nonrotatable, movable, and automatically moving [94]. User-control when moving a robot resulted in a higher social presence than when the robot moved automatically. A small experiment where sliding movements resulted in similar effects on social presence as the forward-backward motion is also reported on in [94]. To clarify the effects of a remote camera's zooming and display's movement on social presence, the work presented in [95] uses results from two experiments that compare (a) relations between the presenter moving and camera zooming (with or without synchronization) and (b) relations between the presenter moving and display moving (with or without synchronization). Via a questionnaire especially developed for the study, the authors found that the zoom caused greater feeling of presence when facing, talking with, and viewing a presenter that moved either in a synchronized or nonsynchronized way with the zoom. On the contrary, the zoom was found to cause reductions in perceived audio and video quality when the presenter was not moving. It was also found that the movement of the display increased the social presence.

The perceived presence during a training session with alarm operators and health care professionals with the Giraff was studied in [72]. The participants followed a realistic scenario in which they "visited an elder" after which they were asked to fill a survey with questions regarding perceived ease of use and presence. The questionnaires used were based on 
the Temple presence inventory [96] and Networked minds social presence inventory [97] typically applied in humancomputer interaction domains which both had been used also in [28]. It was found that the questionnaires were applicable also in the MRP system domain. Correlations were found between how "present" and "attentive" users were during the virtual visit and with how they performed when driving the robot, for example, ease of docking, navigation in the environment [72]. In a further study [75], it was examined how the novice pilot users spatially configured themselves with respect to the elder while being embodied in Giraff. The encoding of spatial formations was done by using the spatial formations defined in the Kendon F-formations system. It was found that some of the novice pilot users also formed another unexpected spatial formation. It was a formation in which the pilot users did not turn Giraff towards the elder in situations where it would be natural to be turned towards each other in a human-human interaction. Further, correlations were found between the participants' perceived presence and how they adhered to the natural spatial configurations when maneuvering the Giraff, thereby suggesting that perceived presence can also be observed by the manner in which the Giraff is being navigated [69].

Using the MeBot platform, [28] measured social presence, trust, cooperation, and engagement and illustrated that social expressions were ranked higher with a gesturing MeBot than with a MeBot not performing any gesturing. In an effort to analyze the interpretability of gestures, facial expressions, and perception of a group discussion, [98] performed two video studies where they found that facial expressions combined with supportive gestures resulted in more correct interpretations of the expressions, a higher confidence in having understood the expressions, and a larger impact of the message than for the case of only facial expressions. The participants considered the collaborator embodied in a static screen without supportive gestures as being less involved in the conversation than a collaborator who could support the facial expressions with gestures. The participants also perceived both the embodied collaborator and the colleagues involved in the interaction as being more composed and involved when the embodied collaborator took a leadership role in the interaction. Spatial location recalling with a similar type of system as MeBot making use of Skype and arms was investigated in [99]. It was found that participants facing a system with verbal location descriptions accompanied by robotic pointing gestures remembered more locations in comparison with participants facing a system that only provided verbal descriptions when the locations were presented nonsequentially.

The effects of varying the visual framing (decoration versus no decoration) of a MRP system and the verbal framing of the pilot (interdependent versus independent performance) in a desert task study were examined in [100]. They found that participants who were informed that their performance would be evaluated as a team with the pilot were producing more in-group behaviors. Contrary to their expectations, they found that visual framing of the MRP system weakened the team cohesion. The dynamics found in the study was found to be different to the one in humancomputer and computer-mediated communication.
According to [38], several factors are needed to experience a location in an immersive way among which manipulation of objects was one of the outlined factors. Also several other factors are needed to experience a location immersive including a wide high-resolution visual field with accuracy, preserved gaze and life-like appearances (i.e. having the same horizontal and vertical visual angles when seem from remote), high-dynamic range of audio with a directional sound field and the ability to move around in the environment [38]. With the goal to provide benefits of physical travel in an immersive way, [38] described a mutually immersive MRP system equipped with arms that would allow pilots to participate in meetings in remote locations.

Also in [101], an attempt to find communication performance measures for evaluating MRP systems was made. The authors provide a list of quantitative measures drawn from fields such as human-computer interaction, computersupported cooperative work, communication, and psychology.

4.2. Attitudes and Acceptance Measures. Attitude measures to robotics have been studied in a variety of domains to uncover differences in attitudes relating to specific technical solutions, or to cultural effects. The applicability of Negative Attitude toward Robots Scale (NARS), originally presented in [102], on MRP systems was studied in [103]. By performing three different studies: (1) video evaluation, (2) pilot a MRP system, and (3) interact with a MRP system, the authors found that NARS may be applied in the MRP system domain. However, [103] suggested that the NARS-S3, which regards the perceived emotions when talking to robots in general, may need to be modified towards less general terms. Also culture, gender, and prior experience to robots were found to influence the NARS score and participants being generally positive to robots were more positive to the MRP systems. Similarly to [92], the authors emphasized the need to complement questionnaires with other methods. They used ethnographic methods such as observations and interviews while claiming that numbers alone can only highlight issues but not explain the reason for them.

Also when measuring attitudes, other parameters have been used as indicators. For example, it is suggested in [104] that eye contact and height are important factors that can have an impact on the attitude. For example, children chose a significantly shorter distance to the $112 \mathrm{~cm}$ tall Mobi (not to be confused with mObi from Bossa Nova Robotics.) Jr. $(26.8 \mathrm{~cm})$ than to the $175 \mathrm{~cm}$ tall Mobi Sr. $(70.4 \mathrm{~cm})$ during interactions observed at an arts and technology festival. There were also significant differences between genders in the teenager and adult groups. Females chose an in average longer distance to the robots than men. Further, it was found of importance that the pilot's face was shown during interaction in [105] in which a robot guided people at an exhibition. The participants faced the robot and interacted longer with the robot when it showed the face of the pilot and subjectively rated the robot with a face higher.

A closed-loop methodology including prototyping, field testing in residential care settings, assessment on, for example, satisfaction, and further development was proposed in 
[71]. The authors reported on focus groups within the ASSIST project that were given a demonstration on a proposed system via video before discussing cost, functionality, interface complexity, and special versus general purpose. In the study, the robot, uBot-4, primarily a research robot, was used as a prototype for mobile manipulation. The uBot- 4 and the later version uBot-5 had an LCD screen which is nonexisting on the uBot-6 [106]. The elderly focus group was positive to video communication technology and stated that impairment would overrun concerns about privacy. They further seemed less afraid of technologies they did not understand when believing the technology could be a benefit to them. Specially appreciated with the methodology was the access to the researchers and their willingness to respond to questions and concerns. This implies that a closed-loop methodology as proposed in ASSIST might improve early adoption.

4.3. Societal and Ethical Issues. There has been a subset of works that are concerned with measuring and reporting on general concerns on the uptake of MRP systems when applied in homes or at hospitals. These issues as well as ethical concerns are typically reported when MRP systems are first introduced or presented.

The awareness of robot applications among medical and health care professionals was examined in [107]. Concerns found were loss of human interaction, replacement of professionals and staff, costs, and health care coverage. The authors wrote that applications could be eased in acceptance by being cost effective in design and implementation, appropriately defining tasks, and increasing knowledge about on-going research and appropriate human-robot interaction. They also discussed how to educate medical and health care students/professionals and suggested cross-publishing in different communities and coverage of both the medical/health and the robotics field at conferences. Similar concerns (i.e. loss of human interaction and replacement of professionals and staff) were found in a video-based evaluation regarding the Giraff system with different groups of primary health care organizations [73]. In this study, the teachers were more positive than students (as in [57]), demonstrating that a greater exposure to technology does not necessarily increase the acceptance. It was also found in this study that there were large variances between different categories of primary caregivers. The study [73] provides a number of suggestions with respect to increasing the acceptance of technology for elderly, for example, early introduction to technology during the education.

In an attempt to determine motives for implementing robotic telemedicine programs in emergency and critical care in North America and Europe, a web-based survey was performed [108]. The results indicate that there are no cultural issues creating barriers which impede acceptance of MRP systems. Similarly to what was reported in [107], factors perceived to impede the uptake of MRP systems relate to regulations and costs. Motives for implementing and maintaining a program are improved quality, filling service gaps, immediate access to patients, provision of clinical support, and addressing patient satisfaction.
Results from focus groups with health care professionals, elderly, and a set of users (e.g. engineers, physiotherapists, and physicians) were presented in [64]. Potential applications for MRP systems included monitoring of autonomy loss and patient abilities, rapid access when released from hospital, and remote training of caregivers. Ethical issues regarding the camera and privacy as well as usability issues regarding size and cost of robot were reported in [65].

How elderly reacted to the Texai was examined in [2]. When being asked with whom they would like to interact, family interactions were the number one motivation for using the system. Concerns raised were mainly due to etiquette, privacy, and misuse of the system.

4.4. Technical Measures. A number of studies have evaluated the technical aspects connected to the robot and the pilot interface. The pilot interface is critical with respect to usability of the system and has been evaluated in terms of effectivness and security of operations, navigation strategies, and mental workload. In addition, specific technical aspects have been studied to assess if and how much the aforementioned aspects contribute to usability. In particular, semi-autonomy has been studied in several settings using different platforms. Finally, an important and yet unresolved issue is the problem of lost WiFi connection.

4.4.1. Pilot Interface Design. Interfaces used to connect to the robot are as critical as the robot design for creating conditions for successful interaction. A number of studies focus primarily on evaluation and interface design for the pilot users. Efficiency and security of operation were limited by interface design in a pilot study on two MRP systems in [63]. The visual information and the control mechanisms impacted performance. In a further study [66], novice pilots performed six different tasks in home-like conditions using three different navigation systems. The compared systems were (1) Video-Centric Display (VC2D), (2) Augmented Reality Display (AR3D), and (3) Mixed Perspective Exocentric Display (ME3D). Particularly for women, people over 30 years of age and those working less than $22 \mathrm{~h}$ a week with computers, the ME3D was the most effective in terms of completion time and quality of commands in moving tasks. The AR3D was preferable in precise navigation tasks. The perceived ease of use and perceived performance were the highest for ME3D and the lowest for VC2D. The results corroborate previous research findings, for example, [109113].

Pros and cons with hand gesturing in comparison with control by a mouse or a joystick was discussed in [114]. Many of the problems were associated with the need to wear a data glove but it was also found that hand gestures were more tireding and required the pilot to remember a set of possible gestures. On the contrary, it was found more easy to use for operations such as grasping.

Navigation strategies were studied with ten rehabilitation professionals when performing a set of navigation tasks in unknown, home-like environments 2-3 weeks after receiving training on a user interface $[67,68]$. It was found that the 
pilots with worse performance used more commands and drove closer to obstacles. Further, the worst pilots needed less assistance due to keeping a lower speed in general. Also the gaze behavior during the navigation was analyzed and the pilots mostly gazed at the radar area. The authors conclude that the radar area seems to have provided the users with useful feedback on distance to objects.

4.4.2. Semi-Autonomy. How semi-autonomous functionalities could be used in assisting driving was examined in [115]. A number of concrete technical solutions were implemented: (1) create a map of recent obstacles and (2) create a trajectory of the free path. Twenty four users were selected to pilot the Texai through an obstacle course. Measured parameters were time of task (completing obstacle course) and number of errors/collisions. It was found that while the assisted teleoperation helped people to avoid obstacles, also the time to complete an obstacle course increased. A guiding principle is to have two different video profiles: one dynamic used during movement and one for stationary use where a higher resolution might be more desired [41]. If sensor information is available, it must be correct and provided with timing. In order not to overwhelm the pilot, only the readings relevant for the pilot should be provided. Most users requested a map in the user interface and a wide-angle web cam that could be panned or tilted. To safely use the robot, autonomous behaviours, such as, follow a person and go to a specified location, were found to be necessary. This in combination with the ability to pan the camera would aid the pilot in walking conversations, a need that is highlighted in [42] where the robot is used in office environments and could be expected to walk at the same speed as local persons.

Semi-autonomous functionalities particularly for the home environment have also been studied. This is particularly useful for novice pilot users such as health care professionals with little exposure to ICT technologies. Three preliminary studies conducted during the production of the first prototype of Telerobot were reported on in [64]. As homes have many physical constraints, for example, doorsteps, doorframes, and carpets, a set of trials were performed exploring different means of navigation. It was noted that position point navigation worked better for untrained pilots while waypoint navigation worked better for trained pilots. Also within the ExCITE project, efforts presented in [116] are made to apply semi-autonomous functions. Based on user feedback via questionnaires and interviews from 15 people having driven at least two different Giraffs multiple times, the authors describe algorithmic solutions to automatic docking, detecting obstacles and self-localization on a map.

Another way to measure how different means for navigation affect the quality of a pilot interface is to measure the mental workload with the NASA TLX test [117]. This measure was used together with the USE Questionnaire [118] in a study in which ten novice participants navigated the Giraff by following a dotted path on the floor via different checkpoints and performing a task received along the track of checkpoints [119].
Presence has also been evaluated in systems with partial autonomy. A system equipped with a semi-autonomous navigation control, semi-autonomous people tracking, and improved situational awareness was compared with a system lacking assisted control in [120]. Using Witmer and Singer's presence questionnaire [121], they found that the user satisfaction was significantly higher for the system with assisted control.

\subsubsection{Communication Channel Reliability and Robustness.} When using a MRP system, the pilot of the system is not at the location of the system and as such the pilot neither can push the system back into areas with WiFi coverage in case of a lost connection nor can ask the local users to push the system back as the connection is lost. This issue was discussed in [122] with the claim that it needs to be addressed before real deployments can be made possible. This is important due to limitations in range of WiFi and also because metal objects such as elevators can cause invisible network shadows. The solution discussed to overcome the problem is reversing the motion in slow speed until sufficient access to the WiFi is recovered. However, the MRP system needs to halt in case it cannot acquire the connection after a limited amount of seconds as the reason may be due to the network. This solution does not work if new obstacles (i.e. doors) have just closed behind them. The authors further suggested that a light or backup beep could be used to indicate the robot's intention particularly when it is being unoccupied.

To achieve a sufficient audio and video quality during interaction through a MRP system, there is again a need for a reliable connection without latencies or high data losses. A number of measures of audio and video quality are suggested in [101]. These include the ITU-T Recommendation P.805 [123] for subjectively measuring the quality of speech, P.910 [124] for subjectively testing the multimedia content, and the Perceptual Evaluation of Video Quality (PEVQ) [125] for objectively measuring video signal quality using simulation tests.

\section{Design Implications for Future MRP Systems}

While each of the different application areas discussed in this paper has their own specific challenges, the results of the evaluations and the current technological trends can be used to outline a set of common design implications for developers of future MRP systems.

One of the key aspects to consider is privacy. It should be possible to see whether there is anyone embodied in the system or if it is unused. Different solutions for the problem exist today; for example, the Texai [12] uses a black screen when the system is not in use. Giraff [9] takes it one step further by directing the screen, and thus the camera, towards the wall while charging the robot. Following the principle of privacy, it should also be possible to identify the person trying to connect or currently embodying the MRP system [3].

Another important consideration is the matter of cost and adaptability to different client user platforms and needs. Today, there are several systems that leverage on the use of the 
open source software Skype and the standard hardware and software for robotics (ROS). It is our belief that MRP systems should support various client platforms.

We have outlined a number of user evaluations and studies on pilot interface design and semi-autonomous functionalities where it was found that assisted driving is preferable, for example, $[87,115,120]$, that a map of the environment is needed, for example, $[41,61,78,116]$, and that means are needed for lowering the burden of the pilots such as obstacle avoidance [115, 116] and assisted docking [116]. However, none of them have considered how to best support a pilot user who connects to a MRP system through a smartphone or tablet device. For pilots using such systems, it is likely to be even more important that the client interface includes a map of the environment on which the pilot can make requests where to go by clicking at different locations as well as help to dock the robots and avoid obstacles.

Already today, several of the existing MRP systems offer an adjustable height $[12,19,20,24]$. The results of [104] support the need for an adjustable height as people of different length preferred robots of different height and adjusted their distance to them accordingly. The need for an adjustable height can be further motivated by the use of MRP systems in office contexts. While a lot of the informal communication occurs in hallways where local users are usually standing or walking, the same local users can be expected to sit around a table during meetings. Similar issues have been raised also from elderly involved in evaluations within the ExCITE project [70].

Finally, a yet unresolved issue is the communication channel robustness and what to do if a connection to the MRP system is lost due to loss of the WiFi connection. Particularly in home environments inhabited by fragile elderly with a low experience of computer technologies, it is important to develop methodologies for how to resolve the issue as the elderly cannot be expected to be able to push the system back to a zone with WiFi coverage or to the docking station. Further, as new technologies for connecting to the internet arise, for example, the $4 \mathrm{G}$, it would be advisable to allow the local user to choose what type of internet connection should be used, for example, a LAN connection with a wireless router or a $4 \mathrm{G}$ dongle.

\section{Future Outlook}

This paper has provided an overview of the literature in the expanding field of social robotic telepresence. The future outlook for the area is promising and there are several events that show an increase in the development and evaluation of mobile robotics telepresence systems. Such events include workshops and dedicated discussion groups. For examples, a panel discussion on Robot Telepresence with representatives from ATR, VGo Communications, Giraff Technologies AB, and Willow Garage was held during the human-robot interaction (HRI) conference 2012. Among the topics discussed was the need to design general and multipurpose MRP systems versus application specific MRP systems. It was argued that the features, interface, and product ID (appearance) are highly domain dependent and thus platforms should be tailored towards their intended use.

An important aspect in MRP system development is to measure the added value of the communication for users when assessing MRP systems. In some cases there is an added value for the pilot user (e.g. a remote worker attending a staff meeting), while in others there is a higher value for the local user (e.g. a sick student being visited by a teacher).

A number of factors need to be considered in the development of the MRP systems including legal issues (who is responsible in case of accidents?) and responsibilities. As health organizations can acquire MRP systems as part of their care processes, it will also be a challenge to overcome initial skepticism when introducing new technologies. Including health care professionals in the development process could be one way to overcome this issue. It was suggested in [126] that 2012 would be a milestone for MRP systems. They hypothesized that there would be hundreds of MRP systems in office environments. In fact a number of new MRP systems have been presented during 2012. While the goal of hundreds of robots deployed in office environments is not unrealistic, it has been suggested by Goldberg in [127] that key bottlenecks for the development of MRP systems are (1) internet and WiFi reliability and (2) the cost for acquiring units that need to be lowered.

Regardless of the market opportunities, evaluation methods of MRP systems will be of growing interest as devices are introduced to new application areas. Further, understanding the requirements for successful interaction via embodiment will be central when guiding the technical development of the physical platform as well as its interfaces. Issues such as semiautonomy, modularization of components, and accessibility of interfaces will become more increasingly important.

\section{Acknowledgment}

This work has been supported by the EU under the Ambient Assisted Living Joint Program-EXCITE Project (AAL-20092-125).

\section{References}

[1] M. Minsky, Telepresence, OMNI Magazine, 1980.

[2] J. M. Beer and L. Takayama, "Mobile remote presence systems for older adults: acceptance, benefits, and concerns," in Proceedings of the 6th ACM/IEEE International Conference on HumanRobot Interaction (HRI '11), pp. 19-26, March 2011.

[3] M. K. Lee and L. Takayama, "“Now, I have a body": uses and social norms for mobile remote presence in the workplace," in Proceedings of the 29th Annual CHI Conference on Human Factors in Computing Systems (CHI '11), pp. 33-42, May 2011.

[4] ExCITE Project, "ExCITE”, August 2012, http://www.exciteproject.eu.

[5] Social Robotic Telepresence, "HRI, 2011 Workshop," August 2012, http://aass.oru.se/ ali/hri2011ws/.

[6] Social Robotic Telepresence, "Ro-Man 2012 Workshop," August 2012, http://aass.oru.se/ ali/srt2012/.

[7] E. Paulos and J. Canny, "Designing personal tele-embodiment," in Proceedings of the IEEE International Conference on Robotics and Automation (ICRA '98), pp. 3173-3178, May 1998. 
[8] E. Paulos and J. Canny, "Social tele-embodiment: understanding presence," Autonomous Robots, vol. 11, no. 1, pp. 87-95, 2001.

[9] Giraff Technologies AB, “Giraff," August 2012, http://www.giraff .org.

[10] Anybots, “QB," August 2012, http://anybots.com.

[11] M. Gibstein, "CES coverage anybots QB telepresence robot hands on," Tech Tracker Tracking Today's Tech, January 12 2011, http://techtrackr.com/2011/01/12/ces-coverage-anybots-qb-telepresence-robot-hands-on/.

[12] Willow Garage, “Texai,” August 2012, http://www.willowgarage .com/pages/texai.

[13] ROS.org, "ROS," August 2012, http://ros.org.

[14] Suitable Technologies, "Suitable Technologies," August 2012, http://suitabletech.com.

[15] VGo Communications, "VGo," August 2012, http://www.vgo .com.

[16] Ryerson University, “PEBBLES," August 2012, http://www.ryerson.ca/pebbles/.

[17] K. M. Tsui, M. Desai, H. A. Yanco, and C. Uhlik, "Exploring use cases for telepresence robots," in Proceedings of the 6th ACM/IEEE International Conference on Human-Robot Interaction (HRI '11), pp. 11-18, March 2011.

[18] VGo Communications, "VGo User Guide v. 1. 5. 0," August 2012, http://www.vgocom.com/sites/default/files/vgo_user_guide_ v1.5.0.pdf.

[19] Mantaro, "MantaroBot," August 2012, http://mantarobot.com.

[20] Double, "Double Robotics," December 2012, http://www.doublerobotics.com/.

[21] mObi, "Bossa Nova Robotics," December 2012, http://www .bnrobotics.com/mobi/.

[22] Gostai, "Jazz Connect," August 2012, http://gostai.com.

[23] Urbiforge.org, "UrbiForge," December 2012, http://www.urbiforge.org.

[24] iRobot Corporation, "iRobot Ava," August 2012, http://www .irobot.com/ava.

[25] 9thSense, “Telo and Helo," August 2012, http://9thsense.com/.

[26] InTouch Health, “RP-7," August 2012, http://intouchhealth.com.

[27] InTouch Health, "FAQ RP-7," August 2012, http://www .intouchhealth.com/ITHFAQs.pdf.

[28] S. O. Adalgeirsson and C. Breazeal, "MeBot: a robotic platform for socially embodied presence," in Proceedings of the 5th ACM/IEEE International Conference on Human-Robot Interaction (HRI '10), pp. 15-22, Osaka, Japan, March 2010.

[29] D. Sakamoto, T. Kanda, T. Ono, H. Ishiguro, and N. Hagita, "Android as a telecommunication medium with a humanlike presence," in Proceedings of the ACM/IEEE Conference on Human-Robot Interaction (HRI '07), pp. 193-200, March 2007.

[30] K. Ogawa, S. Nishio, K. Koda, G. Balistreri, T. Watanabe, and H. Ishiguro, "Exploring the natural reaction of young and aged person with Telenoid in a real world," Journal of Advanced Computational Intelligence and Intelligent Informatics, vol. 15, no. 5, pp. 592-597, 2011.

[31] R. Yamazaki, S. Nishio, K. Ogawa et al., "How does telenoid affect the communication between children in classroom setting?" in Proceedings of the 30th Annual CHI Conference on Human Factors in Computing Systems (CHI '12), pp. 351-366, May 2012.

[32] H. Tobita, S. Maruyama, and T. Kuji, "Floating avatar: telepresence system using blimps for communication and entertainment," in Proceedings of the 29th Annual CHI Conference on
Human Factors in Computing Systems (CHI '11), pp. 541-550, May 2011.

[33] J. M. Lu, C. Lu, Y. Chen, J. Wang, and Y. Hsu, "Tricminia telepresence robot towards enriched quality of life of the elderly," in Proceedings of the Asia Pacific eCare and TeleCare Congress, 2011.

[34] A. Helal and B. Abdulrazak, “Tecarob: tele-care using telepresence and robotic technology for assisting people with special needs," International Journal of ARM, vol. 7, no. 3, pp. 46-53, 2006.

[35] R. S. Fish, R. Kraut, and R. W. Root, "Evaluating video as a technology for informal communication," in Proceedings of the 10th Annual CHI Conference on Human Factors in Computing Systems (CHI'92), pp. 37-48, 1992.

[36] P. J. Hinds and M. Mortensen, "Understanding conflict in geographically distributed teams: the moderating effects of shared identity, shared context, and spontaneous communication," Organization Science, vol. 16, no. 3, pp. 290-307, 2005.

[37] S. Wjittaker, D. Frohlich, and O. Daly-Jones, "Informal workplace communication: what is ti like and how might we support it?" in Proceedings of the 12th Annual CHI Conference on Human Factors in Computing Systems (CHI '94), pp. 131-137, April 1994.

[38] N. P. Jouppi, "First steps towards mutually-immersive mobile telepresence," in Proceedings of the 8th Conference on Computer Supported Cooperative Work (CSCW'02), pp. 354-363, November 2002.

[39] N. P. Jouppi, S. Iyer, S. Thomas, and A. Slayden, "BiReality: mutually-lmmersive Telepresence," in Proceedings of the 12th ACM International Conference on Multimedia, pp. 860-867, October 2004.

[40] N. P. Jouppi and S. Thomas, “Telepresence systems with automatic preservation of user head height, local rotation, and remote translation," in Proceedings of the IEEE International Conference on Robotics and Automation (ICRA '05), pp. 62-68, April 2005.

[41] M. Desai, K. M. Tsui, H. A. Yanco, and C. Uhlik, "Essential features of telepresence robots," in Proceedings of the IEEE International Conference on Technologies for Practical Robot Applications (TePRA '11), pp. 15-20, April 2011.

[42] E. Guizzo, "When my Avatar went to work," IEEE Spectrum, vol. 47, no. 9, pp. 26-50, 2010.

[43] L. Takayama and J. Go, "Mixing metaphors in mobile remote presence," in Proceedings of the Conference on Computer Supported Cooperative Work (CSCW '12), pp. 495-504, 2012.

[44] A. Paepcke, B. Soto, L. Takayama, F. Koenig, and B. Gassend, "Yelling in the hall: using sidetone to address a problem with mobile remote presence systems," in Proceedings of the Symposium on User Interface Software and Technology, pp. 107116, 2011.

[45] T. Mizumoto, K. Nakadai, T. Yoshida et al., "Design and implementation of selectable sound separation on the Texai telepresence system using hark," in Proceedings of the IEEE International Conference on Robotics and Automation (ICRA '11), pp. 2130-2137, 2011.

[46] L. M. Ellison, M. Nguyen, M. D. Fabrizio, A. Soh, S. Permpongkosol, and L. R. Kavoussi, "Postoperative robotic telerounding: a multicenter randomized assessment of patient outcomes and satisfaction," Archives of Surgery, vol. 142, no. 12, pp. 1177-1181, 2007.

[47] L. M. Ellison, P. A. Pinto, F. Kim et al., "Telerounding and patient satisfaction after surgery," Journal of the American College of Surgeons, vol. 199, no. 4, pp. 523-530, 2004. 
[48] A. Gandsas, M. Parekh, M. M. Bleech, and D. A. Tong, "Robotic telepresence: profit analysis in reducing length of stay after laparoscopic gastric bypass," Journal of the American College of Surgeons, vol. 205, no. 1, pp. 72-77, 2007.

[49] Z. J. Daruwalla, D. R. Collins, and D. P. Moore, “'Orthobot, to your station!" The application of the remote presence robotic system in orthopaedic surgery in Ireland: a pilot study on patient and nursing staff satisfaction," Journal of Robotic Surgery, vol. 4, no. 3, pp. 177-182, 2010.

[50] J. B. Petelin, M. E. Nelson, and J. Goodman, "Deployment and early experience with remote-presence patient care in a community hospital," Surgical Endoscopy and Other Interventional Techniques, vol. 21, no. 1, pp. 53-56, 2007.

[51] P. M. Vespa, "Multimodality monitoring and telemonitoring in neurocritical care: from microdialysis to robotic telepresence," Current Opinion in Critical Care, vol. 11, no. 2, pp. 133-138, 2005.

[52] P. Vespa, "Robotic telepresence in the intensive care unit," Critical Care, vol. 9, no. 4, pp. 319-320, 2005.

[53] P. M. Vespa, C. Miller, X. Hu, V. Nenov, F. Buxey, and N. A. Martin, "Intensive care unit robotic telepresence facilitates rapid physician response to unstable patients and decreased cost in neurointensive care," Surgical Neurology, vol. 67, no. 4, pp. 331-337, 2007.

[54] Y. Wang, F. Lai, and P. Vespa, "Enabling technologies facilitate new healthcare delivery models for acute stroke," Stroke, vol. 41, no. 6, pp. 1076-1078, 2010.

[55] K. K. Chung, K. W. Grathwohl, R. K. Poropatich, S. E. Wolf, and J. B. Holcomb, "Robotic telepresence: past, present, and future," Journal of Cardiothoracic and Vascular Anesthesia, vol. 21, no. 4, pp. 593-596, 2007.

[56] J. F. Sucher, S. R. Todd, S. L. Jones, T. Throckmorton, K. L. Turner, and F. A. Moore, "Robotic telepresence: a helpful adjunct that is viewed favorably by critically ill surgical patients," The American Journal of Surgery, vol. 202, no. 6, pp. 843-847, 2011.

[57] C. D. Smith and J. E. Skandalakis, "Remote presence proctoring by using a wireless remote-control videoconferencing system," Surgical Innovation, vol. 12, no. 2, pp. 139-143, 2005.

[58] R. Agarwal, A. W. Levinson, M. Allaf, D. Markov, A. Nason, and $\mathrm{L}$. M. Su, "The roboConsultant: telementoring and remote presence in the operating room during minimally invasive urologic surgeries using a novel mobile robotic interface," Urology, vol. 70, no. 5, pp. 970-974, 2007.

[59] M. Iftikhar, M. J. Majid, M. Muralindran, G. Thayabaren, R. Vigneswaran, and T. T. K. Brendan, "OTOROB: robot for orthopaedic surgeon roboscope: non-interventional medical robot for telerounding," in Proceedings of the 5th International Conference on Bioinformatics and Biomedical Engineering (iCBBE '11), May 2011.

[60] D. Sampsel, G. Bharwani, D. Mehling, and D. Smith, "Robots as faculty: student and faculty perceptions," Clinical Simulation in Nursing, vol. 7, no. 6, pp. 209-218, 2011.

[61] J. Fitzgerald, "After surgery, a robot may be at your side in quest for efficiency, savings, hospital is testing at-home mechanical monitors," Globe Correspondent, December 2011, http://www.highbeam.com/doc/1P2-30289248.html.

[62] S. Coradeschi, "GIRAFFPLUS: combining social interaction and long term monitoring for promoting independent living," in Proceedings of the Medicinteknikdagarna, 2012.

[63] D. Labonte, F. Michaud, P. Boissy, H. Corriveau, R. Cloutier, and M. A. Roux, "A pilot study on teleoperated mobile robots in home environments," in Proceedings of the IEEE/RSJ International Conference on Intelligent Robots and Systems (IROS '06), pp. 4466-4471, October 2006.

[64] F. Michaud, P. Boissy, D. Labonté et al., "Remote assistance in caregiving using telerobot," in Proceedings of the International Conference on Technology \& Aging, 2007.

[65] F. Michaud, P. Boissy, D. Labonté et al., "A telementoring robot for home care," in Proceedings of the International Conference on Technology \& Aging, A. Mihailidis, A. Boger, H. Kautz, and L. Normie, Eds., vol. 21 of Assistive Technology Research Series, pp. 138-145, IOS Press, Amsterdam, Netherlands, 2008.

[66] D. Labonte, P. Boissy, and F. Michaud, "Comparative analysis of 3-D robot teleoperation interfaces with novice users," IEEE Transactions on Systems, Man, and Cybernetics B, vol. 40, no. 5, pp. 1331-1342, 2010.

[67] F. Michaud, P. Boissy, D. Labonté et al., "Exploratory design and evaluation of a homecare teleassistive mobile robotic system," Mechatronics, vol. 20, no. 7, pp. 751-766, 2010.

[68] P. Boissy, S. Brière, H. Corriveau, A. Grant, M. Lauria, and F. Michaud, "Usability testing of a mobile robotic system for in-home telerehabilitation," in Proceedings of the International Conference on Engineering in Medicine and Biology Society (EMBC'11), pp. 1839-1842, 2011.

[69] H. Eftring, The Useworthiness of Robots for People with Physical Disabilities [doctoral dissertation], 1999.

[70] S. Coradeschi, A. Loutfi, A. Kristoffersson et al., "Towards a methodology for longitudinal evaluation of social robotic telepresence for elderly," in Proceedings of the HRI Workshop on Social Robotics Telepresence, pp. 1-7, 2011.

[71] P. Deegan, R. Grupen, A. Hanson et al., "Mobile manipulators for assisted living in residential settings," Autonomous Robots, vol. 24, no. 2, pp. 179-192, 2008.

[72] A. Kristoffersson, S. Coradeschi, A. Loutfi, and K. Severinson Eklundh, "Towards evaluation of social robotic telepresence based on measures of social and spatial presence," in Proceedings of the HRI Workshop on Social Robotic Telepresence, pp. 43-49, 2011.

[73] A. Kristoffersson, S. Coradeschi, A. Loutfi, and K. SeverinsonEklundh, "An Exploratory Study of Health Professionals' attitudes about robotic telepresence technology," Journal of Technology in Human Services, vol. 29, no. 4, pp. 263-283, 2011.

[74] L. Tiberio, L. Padua, A. R. Pellegrino, I. Aprile, G. Cortellessa, and A. Cesta, "Assessing the tolerance of a telepresence robot in users with mild cognitive impairment-a protocol for studying user's physiological response," in Proceedings of the HRI Workshop on Social Robotic Telepresence, pp. 23-28, 2011.

[75] A. Kristoffersson, K. Severinson Eklundh, and A. Loutfi, "Measuring the quality of interaction in mobile robotic telepresence a pilot's perspective," International Journal of Social Robotics, vol. 5, no. 1, pp. 89-101, 2013.

[76] A. Kristoffersson, K. Severinson Eklundh, and A. Loutfi, "Towards measurement of interaction quality in social robotic telepresence," in Proceedings of the Ro-Man Workshop on Social Robotic Telepresence, pp. 24-31, 2012.

[77] Florence, "Florence project," December 2012, http://www .florence-project.eu.

[78] GiraffPlus, “GiraffPlus project,” December 2012, http://giraffplus.eu.

[79] DN, "Roboten Stepan-klassens ljus," January 21 2011, http:// www.dn.se/nyheter/varlden/roboten-stepan-klassens-ljus. 
[80] R. Bloss, "High school student goes to class robotically," Industrial Robot, vol. 38, no. 5, pp. 465-468, 2011.

[81] Cambio, "Robot helps sick teen attend high school," January 25 2011, http://www.cambio.com/2011/01/25/robot-helps-sickteen-attend-high-school.

[82] Singularityhub, "Texas students attends school as a robot-a sign of things to come," February 2 2011, http://singularityhub .com/2011/02/02/texas-student-attends-school-as-a-robot-arobot-a-sign-of-things-to-come-video/.

[83] E. Bridgeman and N. C. Bridgeman, "A future for robots in schools: fact of fantasy?" New Zealand Journal of Applied Computing and Information Technology, vol. 12, no. 1, 2008.

[84] D. I. Fels, J. K. Waalen, S. Zhai, and P. L. Weiss, “Telepresence under exceptional circumstances: enriching the connection to school for sick children," in Proceedings of the International Conference on Human-Computer Interaction (INTERACT '01), pp. 617-624, 2001.

[85] A. Cheetham, C. Young, and D. I. Fels, "Interface development for a child's video conferencing robot," in Proceedings of the IEA2000/HFES2000 Congress, vol. 1, pp. 380-383, 2000.

[86] D. I. Fels and P. L. Weiss, “Toward determining an attentiongetting device for improving interaction during video-mediated communication," Computers in Human Behavior, vol. 16, no. 2, pp. 189-198, 2000.

[87] J. Yeung and D. I. Fels, "A remote telepresence system for high school classrooms," in Proceedings of the Canadian Conference on Electrical and Computer Engineering, pp. 1447-1450, May 2005.

[88] Maastricht University, "Swarmlab robots agents \& interaction," August 2012, http://swarmlab.unimaas.nl/.

[89] D. Schneider, "I, office worker," IEEE Spectrum, vol. 47, no. 10, pp. 20-21, 2010.

[90] N. Karnad and V. Isler, "A multi-robot system for unconfined video-conferencing," in Proceedings of the IEEE International Conference on Robotics and Automation (ICRA '10), pp. 356-361, May 2010.

[91] D. Lazewatsky and W. Smart, "An inexpensive robot platform for teleoperation and experimentation," in Proceedings of the International Conference on Robotics and Automation (ICRA '11), pp. 1211-1216, 2011.

[92] D. Sirkin, G. Venolia, J. Tang et al., "Motion and attention in a kinetic videoconferencing proxy," in Proceedings of the International Conference on Human-Computer Interaction (INTERACT '11), pp. 162-180, 2011.

[93] International Society for Presence Research, "The concept of presence: explication statement," 2000, http://ispr.info/.

[94] H. Nakanishi, Y. Murakami, D. Nogami, and H. Ishiguro, "Minimum movement matters: impact of robot-mounted cameras on social telepresence," in Proceedings of the ACM Conference on Computer Supported Cooperative Work (CSCW'08), pp. 303312, November 2008.

[95] H. Nakanishi, K. Kato, and H. Ishiguro, "Zoom cameras and movable displays enhance social telepresence," in Proceedings of the 29th Annual CHI Conference on Human Factors in Computing Systems (CHI '11), pp. 63-72, May 2011.

[96] M. Lombard, T. B. Ditton, and L. Weinstein, "Measuring presence: the temple presence inventory," in Proceedings of the 12th Annual International Workshop on Presence, 2009.

[97] F. Biocca and C. Harms, "Networked minds social presence inventory: (Scales only, Version 1.2)," December 2012, http:// cogprints.org/6742/1/2002_netminds_scales.pdf.
[98] D. Sirkin and W. Ju, "Communicating meaning and team role through gesturing robots," in Proceedings of RSS Workshop on Human-Robot Interaction: Perspectives and Contributions to Robotics from the Human Sciences, 2011.

[99] J. J. Cabibihan, W. C. So, S. Saj S, and Z. Zhang, "Telerobotic pointing gestures shape human spatial cognition," International Journal of Social Robotics, vol. 4, no. 3, pp. 263-272, 2012.

[100] I. Rae, L. Takayama, and B. Mutlu, "One of the gang: supporting in-group behavior for embodied mediated communication," in Proceedings of the 30th Annual CHI Conference on Human Factors in Computing Systems (CHI '12), pp. 3091-3100, May 2012.

[101] K. M. Tsui, M. Desai, and H. A. Yanco, “Towards measuring the quality of interaction: communication through telepresence robots," in Proceedings of the Performance Metrics for Intelligent Systems, 2012.

[102] T. Nomura, T. Suzuki, T. Kanda, and K. Kato, "Measurement of negative attitudes towards robots," Interaction Studies, vol. 7, no. 3, pp. 437-454, 2006.

[103] K. M. Tsui, M. Desai, H. A. Yanco, H. Cramer, and N. Kemper, "Measuring attitudes towards telepresence robots," International Journal of Intelligent Control and Systems, vol. 16, no. 2, pp. 113-123, 2011.

[104] T. van Oosterhout and A. Visser, "A visual method for proxemics measurements," in Proceedings of the Workshop on Metrics for Human-Robot Interaction, pp. 61-68, 2008.

[105] H. Kuzuoka, Y. Furusawa, N. Kobayashi, and K. Yamazaki, "Effect of displaying a remote operator's face on a media robot," in Proceedings of the International Conference on Control, Automation and Systems (ICCAS '07), pp. 745-748, October 2007.

[106] LPR, "Laboratory for perceptual robotics," August 2012, http://www-robotics.cs.umass.edu/index.php/Robots/Robots.

[107] K. M. Tsui and H. A. Yanco, "Assistive, rehabilitation, and surgical robots from the perspective of medical and healthcare professionals," in Proceedings of the AAAI Workshop on Human Implications of Human-Robot Interaction, pp. 34-39, July 2007.

[108] H. J. Rogove, D. McArthur, B. M. Demaerschalk, and P. M. Vespa, "Barriers to telemedicine: survey of current users in acute care units," Telemedicine and e-Health, vol. 18, no. 1, pp. 43-53, 2012.

[109] D. B. Baker, R. Zhou, and D. Song, "Design and prototyping of an economical teleoperations test-bed for human factors research: cost, resource requirements and capability assessment," in Proceedings of the International Conference on Computers and Industrial Engineering, pp. 526-529, 2005.

[110] D. J. Bruemmer, D. A. Few, M. C. Walton et al., ““Turn off the television!": real-world robotic exploration experiments with a virtual 3-D display," in Proceedings of the 38th Annual Hawaii International Conference on System Sciences, p. 296, January 2005.

[111] J. L. Drury, J. Scholtz, and H. Yanco, "Awareness in human robot interactions," in Proceedings of the IEEE International Conference on Systems, Man and Cybernetics, pp. 568-573, 2003.

[112] B. Keyes, R. Casey, H. A. Yanco, B. A. Maxwell, and Y. Georgiev, "Camera placement and multi-camera fusion for remote robot operation," in Proceedings of the IEEE International Symposium on Safety, Security, and Rescue Robotics, 2006.

[113] B. Ricks, C. W. Nielsen, and M. A. Goodrich, "Ecological displays for robot interaction: a new perspective," in Proceedings of the IEEE/RSJ International Conference on Intelligent Robots and Systems (IROS '04), pp. 2855-2860, October 2004. 
[114] T. Ito, "Hand gesture-based manipulation of a personalized avatar robot in remote communication," in Proceedings of HCII International Conference on Human Interface and the Management of Information, pp. 425-434, 2011.

[115] L. Takayama, E. Marder-Eppstein, H. Harris, and J. Beer, "Assisted driving of a mobile remote presence system; System design and controlled user evaluation," in Proceedings of the International Conference on Robotics and Automation (ICRA '11), pp. 1883-1889, 2011.

[116] J. González-Jiménez, C. Galindo, and J. R. Ruiz-Sarmiento, "Technical improvements of the giraff telepresence robot based on users' evaluation," in Proceedings of the 21st IEEE International Symposium on Robot and Human Interactive Communication (Ro-Man '12), September 2012.

[117] S. G. Hart, "NASA-task load index (NASA-TLX); 20 years later," in Proceedings of the 50th Annual Meeting of the Human Factors and Ergonomics Society (HFES '06), pp. 904-908, October 2006.

[118] A. M. Lund, "Measuring usability with the USE questionnaire," STC Usability SIG Newsletter, vol. 8, no. 2, 2001.

[119] A. Kiselev and A. Loutfi, "Using a mental workload index as a measure of usability of a user interface for social robotic telepresence," in Proceedings of the Ro-Man Workshop on Social Robotic Telepresence, pp. 3-6, 2012.

[120] L. Riano, C. Burbridge, and T. M. McGinnity, "A study of enhanced robot autonomy in telepresence," in Proceedings of the Artificial Intelligence and Cognitive Systems, 2011.

[121] B. G. Witmer and M. J. Singer, "Measuring presence in virtual environments: a presence questionnaire," Presence, vol. 7, no. 3, pp. 225-240, 1998.

[122] N. P. Jouppi, S. Iyer, W. Mack, S. Thomas, and A. Slayden, "A first generation mutually-immersive mobile telepresence surrogate with automatic backtracking," in Proceedings of the IEEE International Conference on Robotics and Automation (ICRA '04), pp. 1670-1675, May 2004.

[123] ITU-T Recommendations, "P. 805 (04/2007) —Subjective evaluation of conversational quality," April 2007, http://www.itu.int/ ITU-T/recommendations/rec.aspx?rec $=9066$.

[124] ITU-T Recommendations, "P. 910 (04/2008) — subjective video quality assessment methods for multimedia applications," April 2008, http://www.itu.int/ITU-T/recommendations/rec.aspx? rec $=9317$.

[125] ITU-T Recommendations, "J.247 (08/2008)—objective perceptual multimedia video quality measurement in the presence of a full reference," August 2008, http://www.itu.int/ITU-T/ recommendations/rec.aspx?rec=9497\&lang=en.

[126] E. Guizzo and T. Deyle, "Robotics Trends for 2012," August 2012, http://spectrum.ieee.org/automaton/robotics/roboticshardware/robotics-trends-for-2012.

[127] E. Guizzo, “Telerobots [turning poing]," IEEE Robotics and Automation Magazine, vol. 18, no. 1, 2011. 

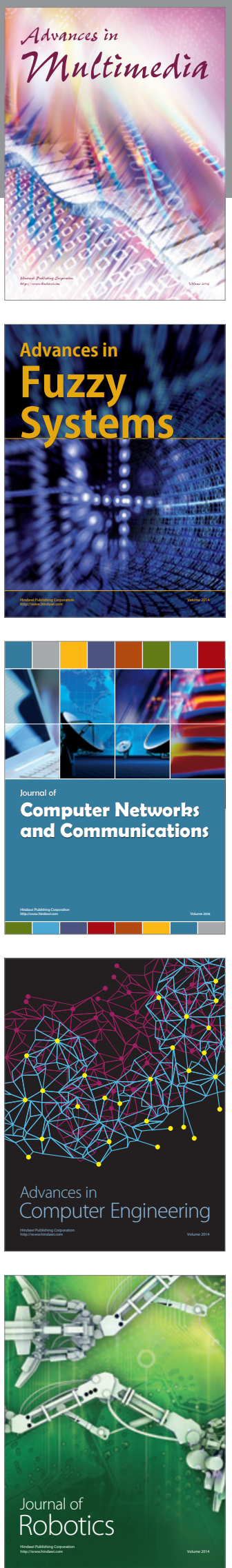

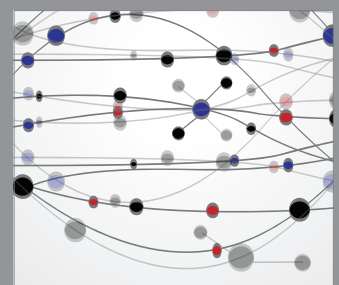

The Scientific World Journal
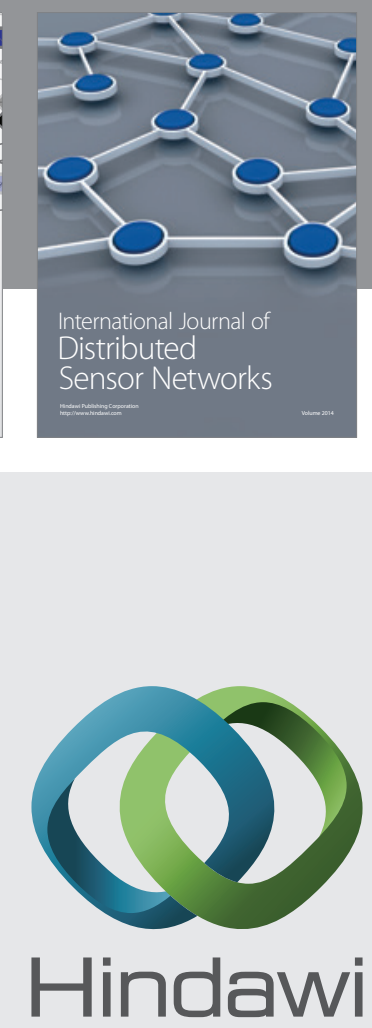

Submit your manuscripts at

http://www.hindawi.com
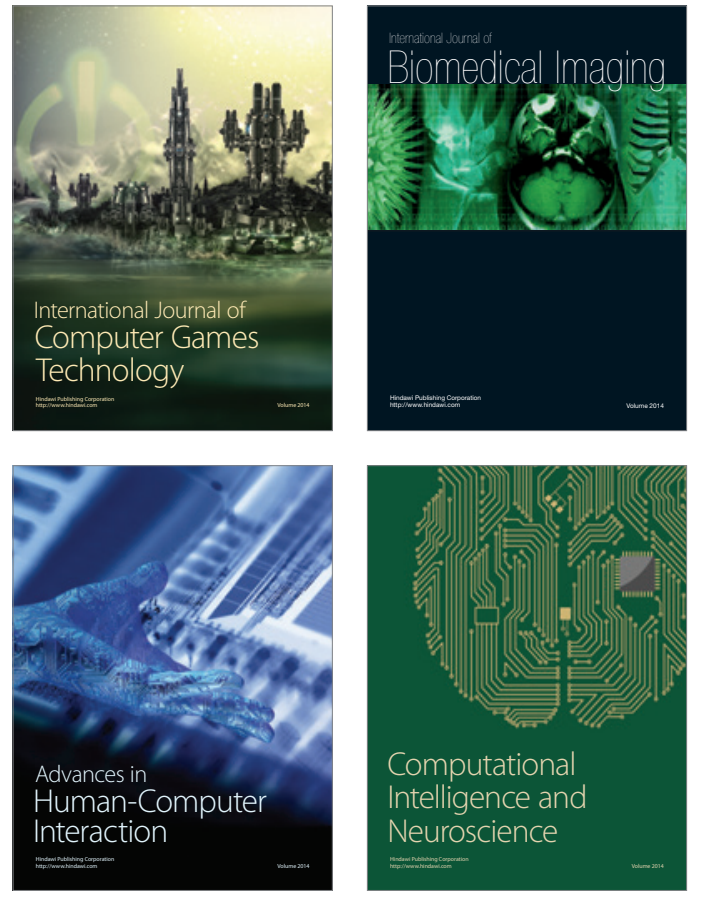
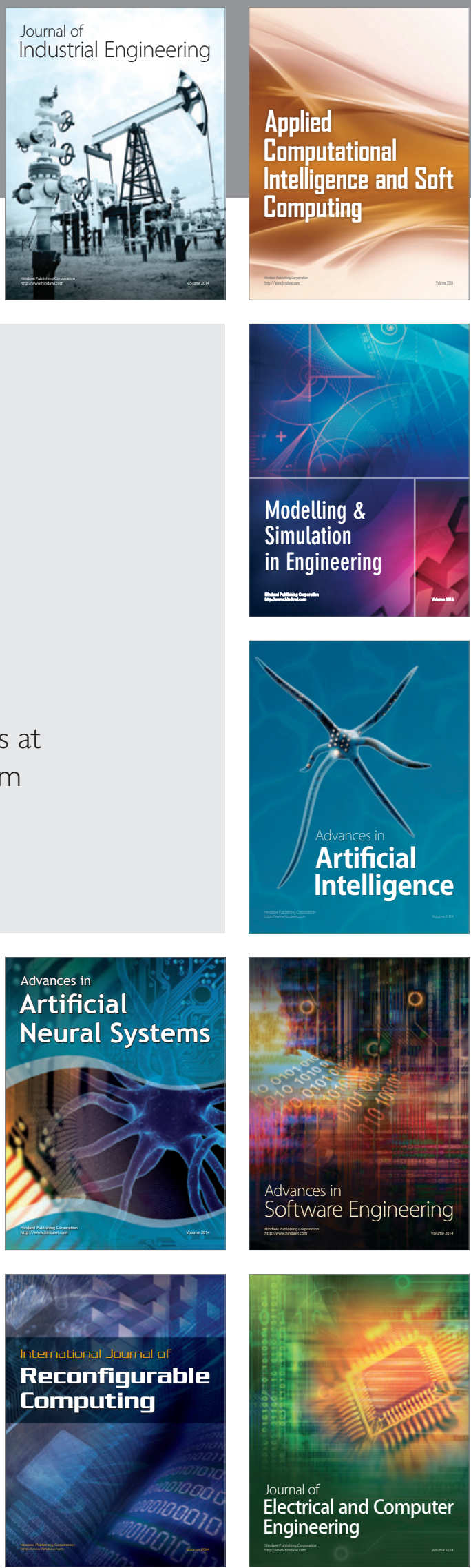